\section{Safe parenteral nutrition and the role of standardised feeds}

JOHN PUNTIS
JOHN PUNTIS $(\square)$

The General Infirmary at Leeds Great George Street Leeds LS1 3EX West Yorks

United Kingdom

Phone: 0441133923828

E-mail: john.puntis@leedsth.nhs.uk

\begin{abstract}
Workload pressure on pharmacies through increased demands for parenteral nutrition (PN) is leading to a growing trend in the use of commercially manufactured PN ('standard feeds') and away from individually 'tailored' prescriptions. This is sometimes justified on grounds of safety, although many areas of risk remain inherent in the process of PN provision. In fact there is little to suggest that widespread introduction of standard feeds would do much to further reduce the already low frequency of serious adverse events. The relative clinical benefits of providing standard feeds or tailored feeds have not been adequately studied, making it impossible to give a clear endorsement of one system over the other. It seems probable that for a proportion of stable patients a range of standard feeds could provide adequate nutritional support, while in unstable patients with complex needs and those needing long term PN, tailored feeds appear the more logical choice. Pharmacy compounding units, therefore, need to remain flexible in their approach to PN provision. Since even small variations in nutrient intake in early life may have long lasting implic ations for extremely premature infants the processes of formulating and providing PN deserve further study.
\end{abstract}

Key words: parenteral nutrition, standard feeds, pre-mixed PN solution, tailored feeds, premature infants

\section{Introduction}

Parenteral nutrition (PN) as a life saving intervention in children was first described forty years ago by Wilmore and Dudrick in an infant with small bowel atresia. (1) Since then it has become widely used for children in whom nutritional requirements cannot be met via the gastrointestinal tract. Although in the child with complex intestinal failure the benefits of PN are unquestionable (e.g. short bowel syndrome), other indications such as premature birth are less well defined. Despite this fact, the premature newborns now comprise one of the largest patient groups to be given PN. (2) Potential advantages of achieving better nutritional support have to be weighed against the risk of complications. In the early years of PN these included serious metabolic disturbance such as lactic acidosis (3) and encephalopathy. (4) With accumulating clinical experience PN has become safer, but major risks remain, including catheter related blood stream infection, (5) intestinal failure associated liver disease (6) and venous thrombosis. (7) The positive effects of a multidisciplinary nutrition support team in reducing the rate of such complications are well recognised. (8)

Other risks lie in the process of providing PN. Adverse events may result from errors in transcription of prescriptions, compounding and administration. For example, inappropriate use (therapeutic risk leading to patient harm), mistakes in diluting concentrated components, complex calculations and manipulations in pharmacy, reconstitution of powders in vials, the need for pumps and syringe drivers, and the use of non-standard giving sets requi- ring light protection and in-line filtration. Greater use of standardised feeds (also known as 'premixed PN solution' or 'commercial PN product') bought in 'ready made' from industrial suppliers, and a reduced reliance on manufacture in hospital pharmacies may be one way of reducing the risk of adverse events. Commercial supply brings with it the reassurance of the feed having been end product tested (although additions such as electrolytes must still be made), something that is not easily achievable in a hospital pharmacy making up individualised feed prescriptions on a daily basis. Pressure to move away from individualised PN prescriptions comes not only from safety concerns but also as a response to rising demand and need to manage pharmacy workload. Safety is rightly a major concern of the clinician, but so too is how to provide the most effective nutritional support for any individual patient. In this respect, the 
relative clinical merits of standardised versus individualised PN prescriptions have been little studied. While recent European guidelines for PN assert that: "uncritical use of standard formulations particularly over a longer period of time may be detrimental to growth and development" (9) there are no good randomised trials to support this expert opinion based statement. To evaluate safety implications further it is necessary to consider where errors do occur in the PN process.

\section{Where do errors in the PN process arise and how might they be mitigated?}

Kuiper et al. (10) reflected on medication errors in general in inpatient pharmacy operations and what might be done to bring about improvements in safety. They pointed out that the inpatient pharmacy component of the medication-use process is complex and error prone, relying on interactions among providers, patients, information and technology. The pharmacists interpret and transcribe prescriptions, prepare them and ensure delivery. Drug preparation and dispensation have been implicated in $11-21 \%$ of all medication errors. PN solutions are complex, containing almost 50 ingredients prepared by the mixing of more than 10 different solutions. Successful technologies reduce the potential for human error by automating tasks that require high levels of accuracy and repetition. A microprocessor-controlled compounding device is an example of such equipment. (11) Risk management strategies remain a fundamentally important adjunct to any such innovation and might, for example, include supervising and regularly performing aseptic validations of pharmacy staff, monitoring for microbial contamination, regular audits, providing standard operating procedures, and enforcing an agreed capacity plan (matching needs to abilities) when necessary. (10)

Sacks et al. (12) explored how often medication errors occurred in relation to $\mathrm{PN}$, as well as the harm that resulted and where in the $\mathrm{PN}$ process errors arose. The setting was a large teaching hospital caring for both children and adults; a prospective study was carried out over a seven month period. A total of 4730 prescriptions for $\mathrm{PN}$ were made in the course of the study; 74 (1.6\%) were associated with a medication error. Of these errors, 1 (1\%) occurred during the prescription process and was detected before preparation; 29 (39\%) occurred during the transcription process, 18 (24\%) during preparation, and 26 (35\%) during the administration process. Sixty seven $(91 \%)$ of the errors were considered as non-harmful whereas $6(8 \%)$ contributed to or resulted in harm to a patient. The rate of errors (15.6/1000 PN days) was similar to that observed in the same institution with 'high risk' drug prescriptions. The authors concluded that errors in the PN process regularly occur and occasionally result in harm, emphasising the need to follow good practice guidelines.

The use of systematic risk analysis methods may be an additional way of improving safety in $\mathrm{PN}$ production. Bonnabry and colleagues (13) write about this approach in their institution where the preparation of paediatric PN included re-transcription and manual compounding of the mixture. Changes to reduce risk were introduced and included new prescription software, direct recording on a server, automatic printing of the labels and piloting of an automatic compounder. They then employed a 'failure modes, effects, and criticality analysis' (FMECA) technique to explore how the safety of the PN process might be improved. FMECA assesses systematically a process or product and enables determination of the location and mechanism of potential failures. A given process is broken down in order to identify possible or likely errors, and gauge what their effect will be, even before they take place. In addition, there is a quantification of failures by a 'criticality index' $(\mathrm{Cl})$ that is calculated by taking into account scores for likelihood of occurrence, severity of the potential effect and chance of detecting the error. The pharmacy team considered 'what could possibly go wrong with this process step?' and Cl was compared between the old and new systems. The new process reduced total $\mathrm{Cl}$ by $59 \%$. The highest risks in the old process were computed for dosage errors, product exchange, failure to detect a dosage/ product error, and product omission. In the new process the most critical steps were labelling mistakes, failure to detect a dosage/product mistake, failure to detect a typing error during prescription, and microbial contamination. The new process significantly increased safety by reducing the risk in administering a solution with a dosage error. The largest improvement in safety was obtained by elimination of fax transmission and re-transcription, and by automation of the compounding procedure. The authors advocate proactive risk analysis methods such as FMECA for improving the safety of high risk processes, especially when it is difficult to directly measure an outcome because of its very low incidence.

\section{Individualised and standar- dised feeds for PN}

Given the heterogeneous nature of patients receiving $\mathrm{PN}$, the possibility of individualising prescriptions taking into account not just age and weight, but variable fluid and electrolyte requirements, partial enteral feeding and additional intravenous fluids seems attractive. Ball et al. (14) described a novel computer assisted prescribing system that could be taken to the bedside and used to come up with a nutritional prescription based on all these factors for each patient. It also included built in safety checks, for example if nutrient intakes were ordered outside set parameters. There are a number of assumptions implicit in the use of such systems: that the pharmacy aseptic compounding unit has the capacity to make up individualised feeds on a daily basis, and that this process is likely to yield clinical benefits compared with using a less flexible standardised feed. It is clear that the first assumption is likely to be challenged in periods of increased demand, and the second 
has not been adequately tested, although some of the relevant literature is discussed below.

Since most PN is provided on a short term basis (for example $1-2$ weeks in the preterm infant) measuring relevant clinical outcomes is difficult and immediately makes comparison of different feeding systems problematical. Cade et al. (15) randomly allocated 52 consecutive preterm infants admitted to a large teaching hospital neonatal intensive care unit (NICU) to either standard PN feed (with flexible electrolyte addition) or individualised feed formulated using the computer program originally developed by Ball. (14) The hypothesis of the study was that computer-assisted prescribing through taking into account the nutritional composition of supplementary fluids would reduce PN feed waste, improve electrolyte balance, and promote growth when compared with the standardised feed approach. In fact, no differences were found between the two groups. The authors concluded that the considerable flexibility afforded by computer assisted prescribing may be of little benefit to most patients, while not discounting the possibility of it conferring advantage in some (e.g. those with unusual fluid and nutrient requirements or during prolonged PN). The same group went on to look in detail at what actually happened when using computer assisted prescribing for patients on the NICU, in terms of how often deviations from a standard feed composition occurred, and whether it was plausible that such deviations were of clinical significance. (16) In all, 148 prescriptions were examined and nutrient content compared with a standard formulation (i.e. regimen based on age and size assuming normal fluid, electrolyte and nutrient requirements). One fifth of the feeds conformed completely to the standard regimen, while $80 \%$ showed some deviation. These deviations were, however, mainly in relation to carbohydrate, sodium and phosphate intake and actually reflected a routine practice on this particular NICU that was at variance with the computer $\mathrm{PN}$ regimens. In other words, if this practice was incorporated within a standardised PN prescription, around two thirds of patients might have received almost identical nutrient intakes whether by individualised computer assisted prescribing or by use of a standardised feed.

In another investigation of preterm infants $<33$ weeks gestation, standardised versus individualised feeds were compared with respect to nutrient intakes achieved and biochemical responses. (17) Two commercially produced standardised PN formulations were used in the study, which included 58 patients. The composition of the individualised PN formulations was determined each morning at the discretion of the attending neonatologist based on a prescribing guideline and current biochemistry results. The two study groups were clinically matched; there were no significant differences in biochemical responses; the standardised PN group received $42 \%$ more amino acids during the first week of life, and $25 \%$ more calcium and phosphate than the individualised PN patients. PN acquisition cost was estimated to be $30 \%$ less with the standardised system.

Lenclen and colleagues (18) changed from individualised to standard PN feeds on their NICU and retrospectively compared 40 infants $<32$ weeks gestational age with respect to nutrient intake and plasma biochemistry. They found that a 20\% higher amino acid intake was achieved over the fist week in the standardised group who were also less likely to need insulin infusions and more likely to have better calcium and phosphate intakes. Similar advantage has been suggested in using standardised PN formulations for paediatric intensive care patients (19) where calcium and phosphate intakes were lower and electrolyte imbalance more common when using individualised prescriptions.

A recent study by Eleni-dit-Trolli and colleagues (20) has suggested that clinical benefits may be derived from early individualised parenteral nutrition prescribing. Once again this was a retrospective study following a change in unit prescribing practice, in a relati- vely small group of 40 preterm infants $<28$ weeks gestation. The comparison is not between individualised and standardised feeds, but between two methods of arriving at an individualised prescription. With the new method applied, the parenteral intake was calculated using spreadsheet software which took into account the desired fluid and nutrient intake, the nutrition provided by partial enteral feeding, and the volume of drugs prescribed. This appears to be a more sophisticated approach to PN prescribing than the earlier method used on this unit, although no details of the latter are provided other than the statement "all individualised PN orders were calculated at the bedside". Whatever changes were actually made, the results indicated an improvement in amino acid and energy intake achieved, shorter time to regain birth weight, and lower risk of severe broncho-pulmonary dysplasia. It is difficult to know how to interpret this study, although it does suggest that fine tuning of PN in individual patients leading to quite small differences in nutrient intake, may have measurable clinical benefit. This could in fact be highly relevant to long term outcomes given such investigations as the Bonn longitudinal study of very low birthweight small for gestational age infants that indicated an effect of energy intake in only the first ten days of life on brain growth and mental development persisting through to adulthood. (21) Similarly, minor differences in enteral feeding of preterm infants in the first few weeks of life have also been shown to exert a striking long term effect. (22)

\section{Conclusions}

The complexities of providing PN mean that adverse events will sometimes occur. Compounding errors are rare, although clinical consequences may be serious, including risk of death. There are many ways of reducing risk such as following best practice guidelines, avoiding unnecessary transcription of prescriptions, using automated compounding machines, and regularly auditing activity including performing 
proactive risk analysis. The impact of end product tested commercial standardised feeds on safety is likely to be small, and does not provide a powerful argument in favour of their use. There may be cost savings involved in the use of standardised feeds, but on this point the literature contains conflicting data (23) and an assessment from industry is that costs are not likely to be lowered and may in fact be increased. (24) It is almost certainly the case that a proportion of patients can be managed perfectly well in terms of nutritional outcomes with a range of standardised feeds. These would most probably be patients without complex fluid, electrolyte and nutrient requirements and needing only short term PN. The proportion of such patients (as opposed to those needing individualised feeds in order to provide optimal achievable nutrient intakes) will vary according to the type of hospital institution and complexity of case mix. The evidence that a PN process involving only use of standardised feeds will produce equivalent clinical outcomes to individualised feed prescription is lacking. This may be particularly important in the preterm newborn infant where the effects of relatively small differences in nutrient intake over the early days of life might only be measurable in terms of long term outcomes rather than short term growth and biochemical monitoring. This underlines both the importance of adequately powered nutritional studies with long term outcome measures, and the ability of hospital compounding units to maintain flexibility with respect to the PN process. This will ensure that standardised feeds are used for those patients who can be effectively managed in this way, while the flexibility of individualised PN prescribing will still be necessary for others in order to best meet their nutritional needs.

\section{ACKNOWLEDGEMENT}

I would like to thank Deirdra Hartigan for commenting on this manuscript.

\section{REFERENCES}

1. Wilmore DM, Dudrick SJ. Growth and development of an infant receiving all nutrients by vein. JAMA 1968;203:860-4.

2. Puntis JWL. Nutritional support in neonatology. In: Sobotka L, editor. Basics in Clinical Nutrition. 3rd ed. Prague: Galen; 2004. p. 425-39.

3. Oliva PB. Lactic acidosis. Am J Med 1970;48:209-25.

4. Johnson JD, Albritton WL, Sunshine P. Hyperammonaemia accompanying parenteral nutrition in newborn infants. J Pediatr 1972;81:154-61.

5. Hodge D, Puntis JWL. Diagnosis, prevention, and management of catheter related blood stream infection during long term parenteral nutrition. Arch Dis Child Fetal Neonatal Ed 2002;87:21-4

6. Kelly DA. Intestinal failure associated liver disease - what do we know today? Gastroenterology 2006;130:S70-7.

7. Peters M, Beath SV, Puntis JWL, John P. Superior vena cava thrombosis causing respiratory obstruction successfully resolved by stenting in a small bowel transplantation candidate. Arch Dis Child 2000;83:163-4.

8. Agostoni C, Axelsson I, Colomb V, Goulet O, Koletzko B, Michaelsen KF, Puntis JWL, Rigo J, Shamir R, Szajewska H, Turck D. The need for nutrition support teams in paediatric units: a commentary by the ESPGHAN Committee on Nutrition. JPGN 2005;41:8-11.

9. Organisational aspects of hospital PN. In: Koletzko B, Goulet O, Hunt J, Krohn K, Shamir R, editors. Guidelines on paediatric parenteral nutrition of ESPGHAN, ESPEN, and supported by the ESPR. J Pediatr Gastroent Nutr 2005;41:63-9.

10. Kuiper SA, McCreadie SR, Mitchell JF, Stevenson JG. Medication errors in inpatient pharmacy operations and technologies for improvement. Am J Health System Pharm 2007;64:955-959.

11. Shah J. Automated dispensing of parenteral nutrition formulations. Hosp Pharm 2003;10:63-5.

12. Bonnabry P, Cingria L, Sadeghipour F, Ing H, Fonzo-Christe C, Pfister RE. Use of a systemic risk analysis method to improve safety in production of paediatric parenteral nutrition solutions. Qual Saf Health Care 2005;14:93-8.

13. Sacks GS, Rough S, Kudsk KA. Frequency and severity of harm of medication errors related to the parenteral nutrition process in a large university teaching hospital. Pharmacotherapy 2009;29:966-74.

14. Ball PA, Candy DCA, Puntis JWL, McNeish AS. Portable bedside microcomputer system for management of parenteral nutrition in all age groups. Arch Dis Child 1985;60:435-9.

15. Cade A, Thorp H, Puntis JWL. Does the computer improve the nutritional support of the newborn? Clin Nutr 1997;16:19-23. 
16. Beecroft C, Martin H, Puntis JWL. How often do parenteral nutrition prescriptions for the newborn need to be individualised? Clin Nutr1999;18:83-5.

17. Yeung MY, Smyth JP, Maheshwari R, Shah S. Evaluation of standardised versus individualized total parenteral nutrition regime for neonates less than 33 weeks gestation. J Pediatr Child Health 2003;39:613-7.

18. Lenclen R, Crauste-Manciet S, Narcy P, Boukhouna S, Geffray A, Guerrault M-N, Bordet F, Brossard D. Assessment of implementation of a standardized parenteral formulation for early nutritional support of very preterm infants. Eur J Pediatr 2006;165:512-8.

19. Krohn K, Babl J, Reiter K, Koletzko B. Parenteral nutrition with standard solutions in paediatric intensive care patients. Clin Nutr 2005;24:274-80.

20. Eleni-dit-Trolli S, Kermovant-Duchemin E, Huon C, Mokthari M, Husseini K, Brunet M-L, Dupont C, Lapillonne A. Early individualised parenteral nutrition for preterm infants. Arch Dis Child Fetal Neonatal Ed 2009;94:152-3.

21. Brandt I, Sticker EJ, Lentze MJ. Catch-up growth and head circumference of very low birthweight, small for gestational age preterm infants and mental development to adulthood. J Pediatr 2003;142:463-8.

22. Lucas A, Morley R, Cole TJ. Randomised trial of early diet in preterm babies and later intelligence quotient. Brit Med J 1998;317:1481-7.

23. Miller SJ. Commercial premixed parenteral nutrition: is it right for your institution? Nutr Clin Pract 2009;24:459-69.

24. Diorio LS, Thomas DL. Premix vs. custom TPN technical paper. Englewood: Baxa Corporation [serial online] 2007 [cited 2010 Jan 20]; Available from: http://www.baxa.com/resources/docs/pressreleases/Premix\%20TPN\%20Technical\%20Paper.pdf 